\title{
Emission of Electromagnetic Waves from Counter-Streaming Region in Magnetized Pair Plasmas
}

\author{
Laboratory for Plasma Astrophysics, Faculty of Engineering \\ Shinji Saito and Jun-ichi Sakai \\ d023008@ems.toyama-u.ac.jp
}

\begin{abstract}
The counter-streaming instability in magnetized electron and positron (pair) plasmas is investigated by using two dimensional, electromagnetic, relativistic Particle-In-Cell (PIC) code. It is shown that the electrostatic waves are strongly generated by the counter-streaming instability in a strongly magnetized pair plasmas $\left(\omega_{c e} / \omega_{p e}=2\right)$. It is also shown that the highfrequency electromagnetic waves are generated in the saturated phase of the electrostatic waves. The plasma maser process that the electrostatic waves can be converted to the high frequency electromagnetic waves could be a possible mechanism for the electromagnetic wave emission in the pulsar magnetosphere.
\end{abstract}

Keywords : emission, electromagnetic waves, pulsar, pair plasma

\section{Introduction}

It is generally believed that the generation of coherent radio emission of pulsars is due to the development of plasma instabilities in the pulsar magnetosphere. The most studied instability is the twostream instability( for a review, Arons 1981, Usov 2002, Melrose 2004). It was suggested long time ago that the process of pair creation near the pulsar surface is strongly nonstationary (Sturrock 1971, Ruderman and Sutherland 1975). Usov (1987) and Ursov \& V. Usov (1988) investigated the two stream instability that may develop due to the nonstationary of the plasma flow. According to this model, pair plasma clouds are injected into the pulsar magnetosphere continuously with small enough intervals. The fast particles that form part of following cloud overtake slower particles that form part of preceding cloud. Then, the condition for the development of two stream instability is created.

The two stream instability in magnetized plasmas has generally electromagnetic nature, when the stream velocity becomes relativistic and the magnetic field intensity is such weak that the characteristic frequency ratio becomes $\omega_{c e}<\omega_{p e}$ (Saito \& Sakai 2004). Therefore, it is referred to the two stream magnetic instability, electromagnetic counter-streaming instability (Kazimura et al. 1998; Haruki \& Sakai 2003), or Weibel type instability when the external magnetic field is absent. Recently Saito \& Sakai (2004) developed the counterstreaming instability with external magnetic field such as $\omega_{c e}>\omega_{p e}$ and showed that the counterstreaming instability changes its character from magnetic to electrostatic nature when the external magnetic field increases, $\omega_{c e}>1.5 \omega_{p e}$. They applied their results to the Gamma-ray bursts with strongly polarized nature (Coburn \& Boggs 2003).

In this paper, we investigate the emission process of high frequency electromagnetic waves from the counter-streaming instability region in magnetized pair plasmas. We pay attention to the counterstreaming instability with the strong external magnetic field $\omega_{c e}>1.5 \omega_{p e}$ parallel to the counterstreaming direction. It is shown that the highfrequency electromagnetic waves are generated in the saturation phase of the electrostatic waves. The emission process of high frequency electromagnetic waves driven from the electrostatic waves could be due to the plasma maser mechanism (Nambu 1983).

The plasma maser process (Nambu1983) is one of mechanisms through which the electrostatic waves can be transformed to the electromagnetic waves. Eda et al. (1996) showed by using two dimensional electromagnetic particle-in-cell (PIC) code that high frequency Langmuir waves can be generated by the plasma maser mechanism from low frequency whistler waves excited by an electron temperature anisotropy in electron-ion plasmas. Gyobu et al. (1999) showed that the high frequency electromagnetic waves can be emitted from Langmuir waves generated by an electron beam instability in pair plasmas. They concluded that the process that converts to the high frequency electromagnetic waves from low frequency waves is the plasma maser. In $\S 2$ we present our simulation model. In $\S 3$ we present our simulation results. In final section we summarize our results. 


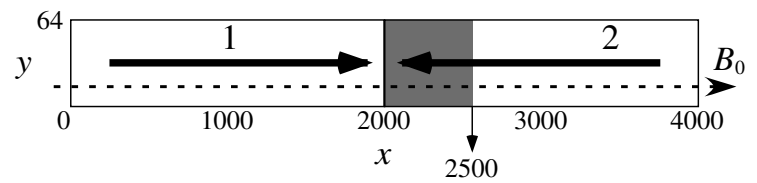

Figure 1: The schematic picture of our simulation model. The notation 1 and 2 means two counterstreaming components. The physical processes in the gray rectangle box are mainly analyzed.

\section{Simulation Model}

We used 2D3V, fully relativistic electromagnetic PIC code, modified from 3D3V TRISTAN code [2]. As shown in Fig.1, the system size is $L_{x}=4000 \Delta$ and $L_{y}=64 \Delta$, where $\Delta(=1$.$) is grid size. The$ periodic boundary conditions for both $\mathrm{x}$ - and $\mathrm{y}$ directions are imposed on particles and fields. There are about 15 million electron-positron pairs, uniformly and keeping the charge neutrality in the system. The average numbers of electron-positron pairs is about 60 per cell. The initial state of the plasma in the left side region $x \leq 2000 \Delta$ has the shifted maxwellian with $v_{d}=+0.5 c$, and in the right side region $x>2000 \Delta$ has that with the $v_{d}=-0.5 c$, where $\mathrm{c}$ is light velocity. This drift velocity parallel to the $\mathrm{x}$-direction corresponds to a Lorentz factor $\Gamma=\left[1-\left(v_{d} / c\right)^{2}\right]^{-1 / 2}=1.15$. The thermal velocity of both electron and positron in whole region is 0.1c. Other parameters are as follows : the time step $\omega_{p e} \Delta t=0.05$, mass ratio $m_{p} / m_{e}=1$., Debye length $v_{t h, e} / \omega_{p e}=1$., the collisionless skin depth $c / \omega_{p e}=10 \Delta$. The external magnetic field is parallel to the counter-streaming direction (x-direction), and the initial electric field and electric current are equal to zero. Other physical quantities like the ratio of $\omega_{c e}$ to $\omega_{p e}$, plasma beta $\beta$, Larmor radius $r_{L}$, and Alfvén velocity $V_{A}$ are $2 ., 0.005,0.5 \Delta$, and $0.81 \mathrm{c}$, respectively.

\section{Simulation Results}

The counter-streaming instability changes its characteristics from magnetic to electrostatic nature when the external magnetic field increases. In this section, we present the time development of the counter-streaming instability with electrostatic nature. Figure 2 shows the spatial distribution of the generated electric fields, both $E_{x}$ (a.1-3) and $E_{z}$ (b.1-3) due to the counter-streaming instability at $\omega_{p e} t=25,50$, and 75 , respectively. The plotted region is a part of right side of the system, where the position $2000 \Delta$ is the center of the
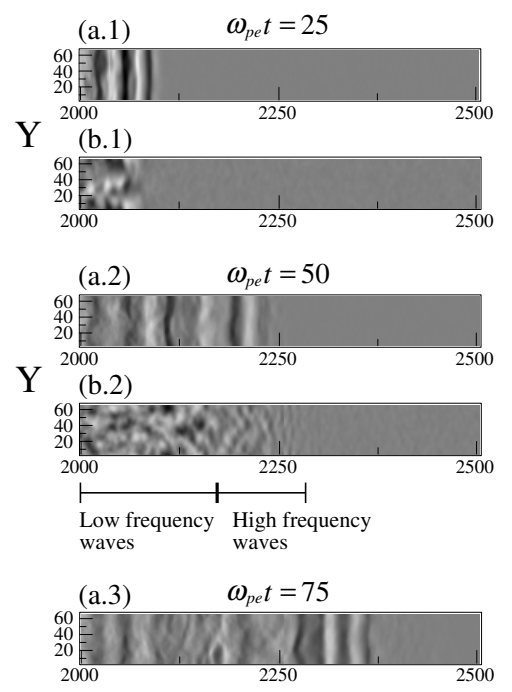

Y (b.3)
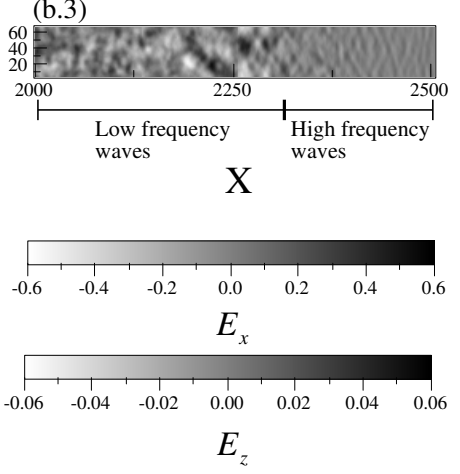

Figure 2: The spatial distribution of the generated electric fields, $E_{x}$ (a.1-3) and $E_{z}$ (b.1-3) at $\omega_{p e} t=25,50$, and 75 , respectively. The plotted region is a part of right side of the system, where the position $2000 \Delta$ is the center of the system.

system (grey region in Fig.1). As seen in Fig.2 (a.1-3), the electrostatic waves are generated due to the counter-streaming, whose propagating velocity is about plasma streaming velocity. The generated electrostatic waves due to the instability can't propagate to the outside of the counter-streaming region, because the generated waves due to the counterstreaming instability have no real part of the frequency. Fig.2 (b.1-3) show the electric field $E_{z}$ of the transverse waves. In the linear stage (until $\omega_{p e} t=20$ ), the electromagnetic waves with small amplitude are generated due to the electromagnetic nature of the instability. These waves also can't propagate from the counter-streaming region to the outside. However, as seen in (b.2) and (b.3), the transverse waves with short wavelength and relatively small intensity propagate to the outside of the counter-streaming region, whose velocity is about 
(a)

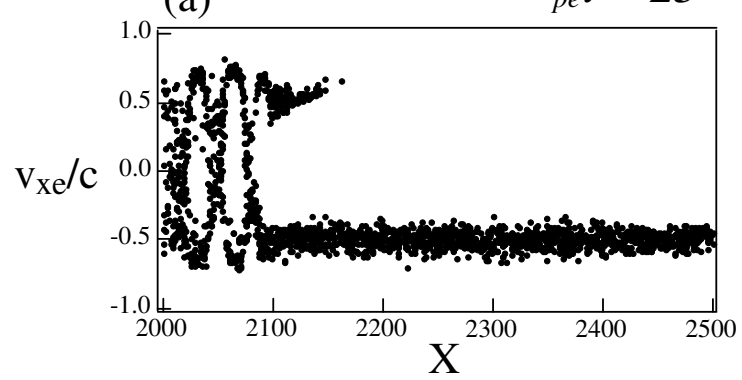

(b)

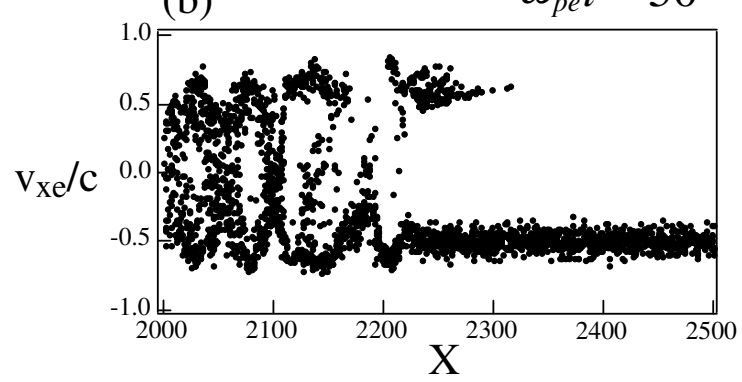

(c)

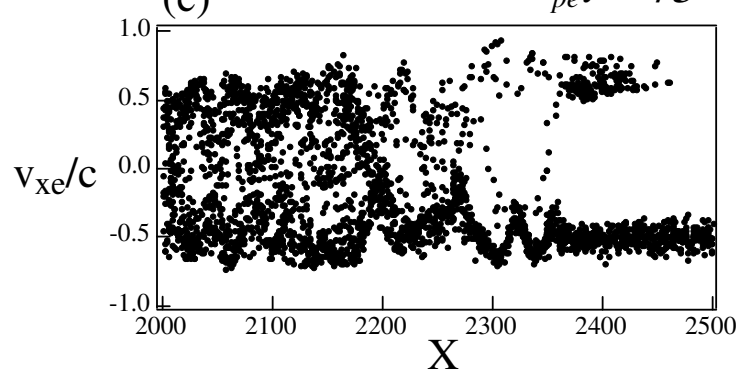

Figure 3: The phase space for electrons at $\omega_{p e} t=(a) 25$, (b) 50, and (c) 75 , respectively.

light velocity. The waves emitted from counterstreaming region certainly differs from the electromagnetic waves generated at the linear stage.

In order to investigate the generation process of the electrostatic waves, in Figure 3 (a), (b), and (c) we show the phase space for the electrons at $\omega_{p e} t=25$, 50, and 75, respectively. The horizontal and vertical axis show the space along external magnetic field between $2000 \Delta$ and $2500 \Delta$, and electron velocity normalized by light velocity along the magnetic field, respectively. At $\omega_{p e} t=25$, the electrostatic waves are generated by the counterstreaming, so that the hole structures appear due to the waves. Then, some electrons at the front of the waves are successively accelerated, resulting in a new state that is unstable to the beam instability. We note here that some positrons are also accelerated with the electrons. Therefore, as seen in (a)
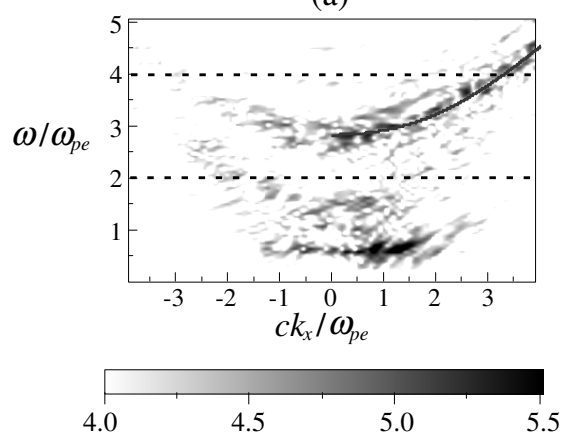

(b)

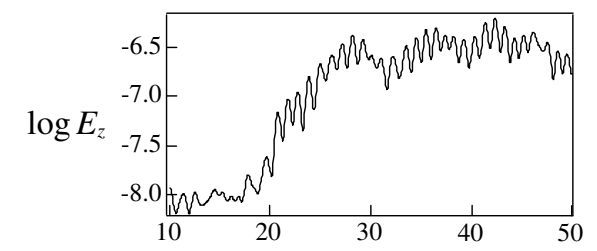

(c)

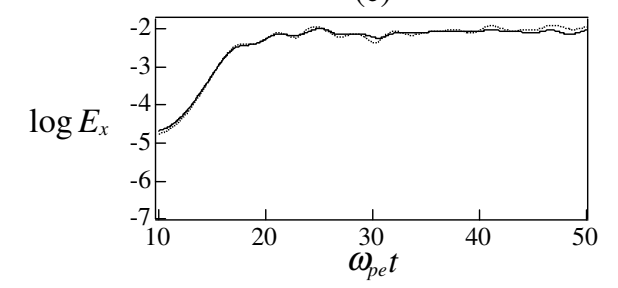

Figure 4: (a) The dispersion relation for $E_{z}$ component on $y=32 \Delta$, by using two dimensional Fourier transformation with both the time between 0 and 2048, and space (x direction) between $2000 \Delta$ and $3024 \Delta$. The solid curve shows the theoretical dispersion curve derived from cold plasma approximation. (b) The time history of $\log \iint E_{z}^{2} d k d \omega$, that is calculated by inverse Fourier transformation with the frequency between two dashed lines in Fig.4(a). (c) The time history of $\log \iint E_{x}^{2} d x d y$, that shows the excitation of the electrostatic waves.

Fig.2(a.3) there appear new electrostatic waves in the region of $x>2250 \Delta$.

Figure 4 (a) shows the dispersion relation for $E_{z}$ component perpendicular to the background magnetic field, by using two dimensional Fourier transformation with both the time between 0 and 2048, and space (x direction) between $2000 \Delta$ and $3024 \Delta$. The horizontal and vertical axis show the wave number normalized by electron skin depth and frequency normalized by electron plasma frequency, respectively. The solid lines show the theoretical dispersion relation of left (or right) polarized waves (high frequency branch) that is derived from cold plasma approximation. The high frequency electromagnetic waves propagate to $+\mathrm{x}$ direction which corresponds 
to the direction of accelerated beam. Figs.4(b) and (c) show the time history of $\log \iint E_{z}^{2} d k d \omega$, and $\log \iint E_{x}^{2} d x d y$, respectively. Fig.4(b) is calculated by inverse Fourier transformation with the frequency between two dashed lines in Fig.4(a), which contain the excited high frequency electromagnetic waves propagating to the direction along external magnetic field. As seen in Figs.4(b) and (c), there is a time lag in the generation of the high frequency electromagnetic waves compared with the electrostatic waves. The electromagnetic waves are generated in the saturated phase of the generated electrostatic waves. That implies that the electromagnetic waves may be generated by some nonlinear conversion process from the electrostatic waves.

\section{$4 \quad$ Summary and Discussions}

We investigated the emission of high frequency electromagnetic waves from the counter-streaming plasma along the external magnetic field. In the strong external magnetic field, the wave generation due to the counter-streaming instability changed its character from the electromagnetic to electrostatic nature. Due to the electrostatic counter-streaming instability, electrostatic waves are generated parallel to the streaming direction. Then, the particles in the front of the waves are accelerated to the relativistic velocity as the beam. It was shown that the high frequency electromagnetic waves are generated in the saturation phase of the electrostatic waves. There is no nonlinear wave coupling such as the three waves interaction between high and low frequency waves.

The plasma maser mechanism leading to the emission of high frequency electromagnetic waves is possible under this condition, as shown by Gyobu et al. (1999). They showed that the electrostatic plasma waves generated from electron beam instability in pair plasmas can be converted to electromagnetic waves through the plasma maser process. We expect that this emission process may be one of important mechanisms for the emission of electromagnetic waves from the pulsar magnetosphere where the electrostatic counter-streaming instability may occur due to the interaction of pair plasma clouds.

\section{References}

[1] Arons, J. 1981, ApJ. 248, 1099

[2] Buneman, O. Computer Space Plasma Physics, Simulation Techniques and Software (Terra Scientific, New York, 1993)

[3] Coburn, W., \& Boggs, S.E. 2003, Nature 423, 415
[4] Eda, M., Sakai, J. I., Zhao, J., Neubert, T., \& Nambu, M. 1996, J. Phys. Soc. Jpn. 24, 916

[5] Gyobu, D., Sakai, J. I., Eda, M., Neubert, T., \& Nambu, M. 1999, J. Phys. Soc. Jpn. 68, 471

[6] Haruki, T., and Sakai, J. I., 2003, Phys. Plasmas 10,392

[7] Kazimura, Y., Sakai, J. I., Neubert, T., \& Bulanov, S. V., 1998, ApJ. Lett. 498, L183

[8] Melrose, D. 2004, D in Young Neutron Stars and Their Environments, IAU Symposium, Vol.218, F.Camilo and B.M. Gaensler, eds.

[9] Nambu, M., 1983, Laser Part. Beams 1, 427.

[10] Ruderman, M.A. \& Sutherland, P.G. 1975, ApJ. 196, 51

[11] Saito, S., \& Sakai, J.I. 2004, Phys. Plasmas in press

[12] Sturrock, P.A. 1971, ApJ. 164, 529

[13] Usov, V.V. 1987, ApJ. 320, 333

[14] Usov, V.V. 2002, proceeding of the 270. WFHeraeus Seminar on: "Neutron Stars, Pulsars and Supernova Remnants" , MPE Report 278, 240

[15] Ursov, V. N. \& Usov, V. V. 1988, Ap\&SS, 140, 325 